\title{
Size is everything
}

DOI:

10.1038/nrm2285

How is tissue size regulated? This question has been occupying scientists for a long time, and although a few genetic regulators of organ size have been identified in organisms such as Drosophila melanogaster, the regulation of organ size in mammals is still perplexing. Do YAP and Yorkie hold the key?

Yorkie (YKI) is a transcriptional co-activator involved in the Hippo ( $\underline{\mathrm{HPO}})$ kinase pathway that suppresses tissue growth in Drosophila. Its mammalian homologue is YAP. The Hippo pathway, which is thought to be conserved in mammals, was previously shown to antagonize the function of YKI. Duojia Pan and colleagues investigated how this is achieved. By using an epitope-tagged

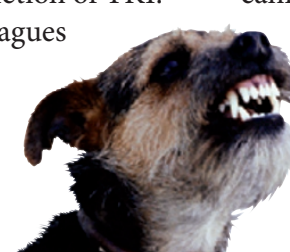

version of YKI in Drosophila cells in vitro, the authors showed that an

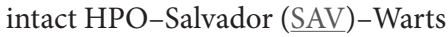
(WTS) pathway resulted in YKI phosphorylation and exclusion from the nucleus. YAP was reported to be phosphorylated by AKT at S127, and a conserved site is present in YKI at S168. Given that WTS belongs to the same kinase family as AKT, it seemed plausible that WTS could phosphorylate YKI. Several in vitro and in vivo experiments indicated that S168 is the main site through which WTS regulates YKI. For example, conditional expression of a YKI S168A mutant protein that cannot be phosphorylated resulted in overgrowth of the eye and lethality in some flies, and disruption of the Hippo pathway resulted in nuclear accumulation of YKI.

Therefore, the Hippo pathway regulates tissue growth by phosphorylating YKI at S168 and excluding it from the nucleus.

The authors then used the similarity between the regulation of YKI and YAP to further map the Hippo pathway in mammals. They found that phosphorylation of S127 by the Hippo pathway also regulated the nuclear localization of YAP in vitro. So can YAP regulate organ size? To answer this, Pan and colleagues made a tetracycline-regulatable Yap transgene that was only expressed in the mouse liver. These mice had much larger livers than controls owing to increased cell proliferation rather than an increase in cell size. Moreover, this overgrowth was dependent on YAP because the livers returned to normal size when the expression of the transgene was turned off. Microarray analyses indicated that YAP induces the expression of genes associated with proliferation and genes known to suppress apoptosis, a combination that proved to be tumorigenic in these mice.

An examination of human cancer cell lines showed that YAP was overexpressed in many of them. However, further work is needed to establish how YAP function is disrupted in human tumorigenesis. The conserved nature of these pathways in flies and mammals should aid in fully understanding the function of the Hippo pathway in the regulation of organ size and tumour development.

\section{Nicola McCarthy, Senior Editor,} Nature Reviews Cancer

ORIGINAL RESEARCH PAPER Dong J. et al. Elucidation of a universal size-control mechanism in Drosophila and mammals. Cell 130 1120-1133 (2007) FURTHER READING Saucedo, L. J. \& Edgar, B. A. Filling out the Hippo pathway. Nature Rev. Mol. Cell Biol. 8, 613-621 (2007) 